\title{
New Design of a Variable Impedance Based on Polarized Diodes at Microwave Frequency
}

\author{
Alejandro J. Venere, Martín Hurtado, Ramón Lopéz La Valle, and Carlos H. Muravchik
}

\begin{abstract}
In this letter, we present a new variable impedance termination consisting of two polarized diodes. To generate a complex-valued impedance, two controlled current sources polarize the diodes, which are interconnected with a Wilkinson power divider through transmission lines of different electric lengths. Because of the reduced number of diodes, this structure presents low power consumption and simple control. We validated the proposed design by implementing a prototype at the operating frequency of 1575.42 MHz. Mapping the measured results in the Smith chart, the impedance can produce reflection coefficients with full-phase coverage within the circle of magnitude 0.35 over a frequency range of $1.5-1.6 \mathrm{GHz}$.
\end{abstract}

Index Terms-One-port impedance termination, p-i-n diode, programmable load, variable impedance.

\section{INTRODUCTION}

$\mathbf{V}$ ARIABLE impedances have been widely used in many problems related to communication systems, including electronically controlled attenuators [1] and modulators [2], phase shifters [3], tunable matching networks [4], adaptive radar absorbers [5], and six-port modulators [6].

A common approach to implement a variable impedance is to exploit the nonlinear relationship between voltage and current in a semiconductor junction. For instance, in [7], a voltage controlled resistance was built using a Schottky diode in forward bias. Because the device has a single degree of freedom, its value is restricted to a line in the complex plane of the reflection coefficient. In [8], a programmable load is generated by an array of p-i-n diodes. The diodes are arranged in cascade to form a transmission line of onehalf wavelength. Using two current sources multiplexed to any diode pair, the impedance reaches full-phase coverage in the Smith chart, while the amplitude of the coverage increases with the number of diodes. Because of the diode array and the multiplexers, this approach results somewhat cumbersome. A simpler architecture is presented in [9], which consists of four varactor diodes and a $3-\mathrm{dB}$ coupler. Four tuning voltages can yield impedance values whose reflection

Manuscript received November 4, 2016; revised January 18, 2017; accepted February 3, 2017. This work was supported in part by the ANPCyT under Grant PICT-2014-1232, in part by the National University of La Plata 1900 under Grant I-209, and in part by the CIC-pBA under Grant 1266/14. (Corresponding author: Alejandro J. Venere.)

A. J. Venere, M. Hurtado, and C. H. Muravchik are with the Research Institute of Electronics, Control and Signal Processing-LEICI, National University of La Plata, La Plata 1900, Argentina (e-mail: alejandro.venere@ing.unlp.edu.ar).

R. Lopéz La Valle is with the Department of Electrical Engineering, National University of La Plata, La Plata 1900, Argentina.

Color versions of one or more of the figures in this paper are available online at http://ieeexplore.iee.org.

Digital Object Identifier 10.1109/LMWC.2017.2690877

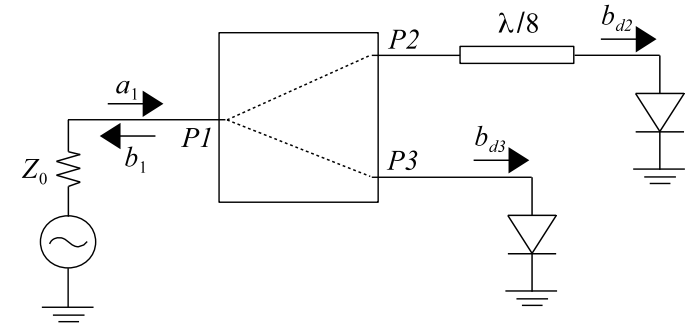

Fig. 1. Basic configuration of the proposed variable impedance.

coefficient reaches the magnitude of nearly 0.9. Additionally, it presents low power consumption given the reduced number of diodes.

In this letter, we propose an alternative design of a variable impedance. The termination load consists of a Wilkinson power divider connecting two p-i-n diodes through transmission lines of different electric lengths. By minimizing the number of diodes required to generate a complex-valued impedance, we simplify the control and reduce the power consumption of the device. Nevertheless, because of the divider, the theoretical value of the variable impedance can achieve complete phase coverage within a circle of normalized radius no larger than 0.5 in the Smith chart.

\section{VARIABle IMPEDANCE DESIGN}

The variable impedance presented in this letter is composed by only two p-i-n diodes whose input impedances are controlled via their polarization currents. The $\mathrm{p}-\mathrm{i}-\mathrm{n}$ junction is an attractive choice because of the large range of its input resistance. The diodes are interconnected through a Wilkinson power divider, as shown in Fig. 1, where port P1 of the divider represents the input port of the impedance termination.

\section{A. Diode Input Impedance}

The $\mathrm{p}-\mathrm{i}-\mathrm{n}$ diode is a $\mathrm{p}-\mathrm{n}$ junction semiconductor diode at low frequency; however, it behaves as a variable resistance at microwave frequencies. A simple forward-biased p-i-n diode model is [10]

$$
Z=R(I)+j \omega L
$$

where $\omega$ is the angular frequency of the signal source, $L$ is the package parasitic inductance, and $R$ is the dynamic resistance of the junction, which depends on the bias current $I$ as $R(I)=K / I^{x}$. The constants $K$ and $x$ are fitting parameters. The parasitic capacitance of the diode has a small influence on the impedance (1) at the frequency band of our interest; hence, 
its effect can be neglected. However, as frequency increases, its effect becomes relevant, limiting the range of $R$. Then, the reflection coefficient at each diode with respect to the characteristic impedance $Z_{0}$ of the transmission line is

$$
\Gamma_{d}(I)=\frac{R(I)+j \omega L-Z_{0}}{R(I)+j \omega L+Z_{0}} .
$$

\section{B. Reflection Coefficient at the Variable Impedance}

When there exists an incident wave $a_{1}$ on port P1 of the Wilkinson power divider, the waves that reach both diodes are

$$
\begin{aligned}
& b_{d 2}=s_{21} e^{-j \phi_{2}} a_{1} \\
& b_{d 3}=s_{31} a_{1}
\end{aligned}
$$

where the coefficients $s_{i j}$ are the scattering parameters of the power divider and $\phi_{2}=\pi / 4$ is the phase shift introduced by the electric length difference of $\lambda / 8$ between the two branches of the variable impedance. Due to the isolation provided by the Wilkinson power divider, there is no coupling between ports $\mathrm{P} 2$ and $\mathrm{P} 3$, then $s_{23}=s_{32}=0$. Because of the mismatch produced by the diodes, the waves $b_{d 2}$ and $b_{d 3}$ are reflected backward and combined to form the total output wave $b_{1}$

$$
b_{1}=s_{12} e^{-j \phi_{2}} b_{d 2} \Gamma_{d}\left(I_{2}\right)+s_{13} b_{d 3} \Gamma_{d}\left(I_{3}\right)
$$

where $I_{2}$ and $I_{3}$ are the forward bias currents applied to each diode. Considering that the Wilkinson power divider is a reciprocal network $\left(s_{i j}=s_{j i}=(-j / \sqrt{2})\right.$ ), when (3) and (4) are replaced in (5)

$$
\begin{aligned}
b_{1} & =s_{21}^{2} e^{-j 2 \phi_{2}} \Gamma_{d}\left(I_{2}\right) a_{1}+s_{31}^{2} \Gamma_{d}\left(I_{3}\right) a_{1} \\
& =\left[j \frac{\Gamma_{d}\left(I_{2}\right)}{2}-\frac{\Gamma_{d}\left(I_{3}\right)}{2}\right] a_{1} .
\end{aligned}
$$

Then, the ratio of $b_{1}$ and $a_{1}$ represents the refection coefficient due to the mismatch between the variable impedance and the reference impedance $Z_{0}$

$$
\begin{aligned}
\Gamma_{1}\left(I_{2}, I_{3}\right) & =j \frac{\Gamma_{d}\left(I_{2}\right)}{2}-\frac{\Gamma_{d}\left(I_{3}\right)}{2} \\
& =\rho\left(I_{2}, I_{3}\right) e^{j \phi\left(I_{2}, I_{3}\right)}
\end{aligned}
$$

where $\rho$ and $\phi$ are the magnitude and phase angle of the reflection coefficient at port $\mathrm{P} 1$.

Neglecting the inductance $L$ of the diode, the reflection coefficient $\Gamma_{d}$ is real valued and ranges about $(-1,1)$ when the bias current $I$ varies from full forward bias to zero. Therefore, the currents $I_{2}$ and $I_{3}$ would control the imaginary and real parts of $\Gamma_{1}$, respectively. Ideally, the proposed variable impedance would produce a reflection coefficient whose magnitude is as large as $\rho=0.707$. Nevertheless, this maximum value of $\rho$ is only possible for a limited number of phase angles $\phi$. Complete coverage of the phase angle $\phi$ is possible for a reflection coefficient with a magnitude of $\rho \leq 0.5$, as shown by the dotted line in Fig. 2(a).

The parasitic inductance of each diode deflects the coverage area of the variable impedance. Then, the complete phase coverage is reduced to a circle of smaller radius $\rho$, as shown by the dotted line in Fig. 2(b). In Section II-C, a method to compensate the inductance $L$ is discussed.

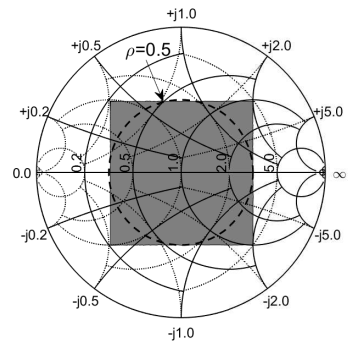

(a)

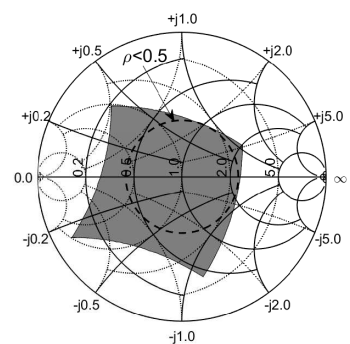

(b)
Fig. 2. Impedance values mapped on the Smith chart. Simulation results of the theoretical model in (8) varying the bias currents $I_{2}$ and $I_{3}$ in the range of $[0,40] \mathrm{mA}$. (a) Neglecting the parasitic inductance $(L=0 \mathrm{H})$. (b) With the parasitic inductance $(L=1.5 \mathrm{nH})$.

\section{Parasitic Inductance Compensation}

To compensate the parasitic inductance $L$ of each diode, we add a reactive element $L_{c}$ connected in parallel to the line at a distance $\lambda / 4$ from the diode, as shown in Fig. 3. Depending on the operating frequency, the compensation can be a lumped element or an open- or short-circuited stub. We could also compensate the parasitic inductance by introducing a series capacitance at the input of the diode. However, we choose the parallel approach, because it is easier to mount on a microstrip board.

Considering that the line section of length $\lambda / 4$ operates as an impedance inverter, the admittance at the position of the compensation is

$$
Y=\frac{Z}{Z_{0}^{2}}
$$

where $Z$ is the diode input impedance given by (1). In order to cancel the imaginary part of (9), the shunt element must be an inductor whose value is

$$
L_{c}=\frac{Z_{0}^{2}}{\omega^{2} L} .
$$

To compute the value of the compensation inductor $L_{c}$, we simulated the admittance in (9) using the S-parameters provided by the manufacturer of the diode.

\section{RESULTS}

This section describes the schematic and its implementation. In addition, it presents measurements recorded from a prototype of the proposed variable impedance.

\section{A. Circuit Implementation}

As shown in Fig. 3, the currents $I_{2}$ and $I_{3}$ that control the impedance are applied to the diode anodes, through the chokes $L_{\mathrm{CH}}$, while the diode cathodes are grounded. The capacitors $C_{\mathrm{DC}}$ appear as an open circuit for the bias currents, but as a short circuit for the microwave signal. The transmission lines of length $\lambda / 4$ and the inductors $L_{c}$ compensate the parasitic inductance of the diodes. In order to generate the real and imaginary parts of the reflection coefficient, the difference length between the two branches is $\lambda / 8$.

We implemented a prototype of the proposed impedance termination by using two p-i-n diodes BAP70-03 of 


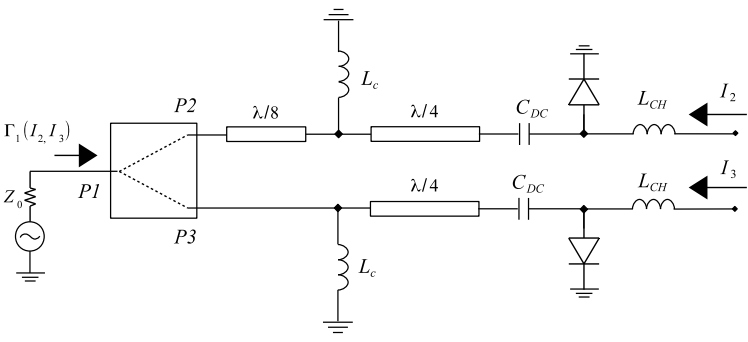

Fig. 3. Schematic of the proposed variable impedance.

NXP Semiconductors and two digitally controlled current sources ADN8810 of Analog Devices. For this prototype, a computer controls the current sources transmitting 12-b words through an serial peripheral interface. The currents can vary between 0 and $40 \mathrm{~mA}$ with a resolution of $\Delta I=9.76 \mu \mathrm{A}$. The settling time of the sources is $3 \mu \mathrm{s}$, which constrains the rate of change of the impedance value. The circuit was implemented on an FR-4 substrate of relative permittivity 4.6 and thickness $1.542 \mathrm{~mm}$. The characteristic impedance of transmission lines is $Z_{0}=50 \Omega$ at the operating frequency of $1575.42 \mathrm{MHz}$. The simulations showed that the imaginary part of the admittance in (9) is $\operatorname{Im}\{Y\}=9 \mathrm{mS}$; then, the compensation inductance must be $11.2 \mathrm{nH}$, but we used a commercial value of $L_{c}=11 \mathrm{nH}$. Other components are $L_{\mathrm{CH}}=33 \mathrm{nH}$ and $C_{\mathrm{DC}}=180 \mathrm{pF}$.

\section{B. Measurement Results}

We first measured the reflection coefficient at the input of the former prototype before adding the compensation. The bias currents $I_{2}$ and $I_{3}$ were swept in the range of $[0,40] \mathrm{mA}$, taking 20 logarithmically spaced values per current source. The coverage area of the proposed controlled impedance without compensating the parasitic inductance $L$ is shown in Fig. 4(a), in blue. Notice that the magnitude of the reflection coefficient can reach at most $\rho=0.22$ for every phase angle (a larger $\rho$ is only possible for limited angles).

After we mounted the compensation $L_{c}$ on the circuit, the coverage area of the variable impedance is closer to the ideal result, as shown in pink in Fig. 4(a). The unknown dielectric loss of the FR-4 could be the reason of the limited improvement produced by the compensation. Nevertheless, the magnitude of the reflection coefficient extends up to $\rho=0.35$ for every phase angle.

In addition, we measured the reflection coefficient of the impedance at different frequencies, as shown in Fig. 4(b). Using the same bias currents as in the former measurement, we recorded the reflection coefficients in the frequency range of 1.5-1.6 GHz. We remark that the variable impedance is still generating a reflection coefficient, whose magnitude is as large as $\rho=0.35$ for any possible phase, since the coverage area only rotates as the frequency changes. Finally, we analyzed the sensitivity of the reflection coefficient in (8) with respect to the parameters $K$ and $x$ that determine the dynamic resistance of the diodes. For the diode BAP70-03, their nominal values are $K=80$ and $x=0.86$, when the units of the bias current are $\mathrm{mA}$. When sweeping those parameters in the range

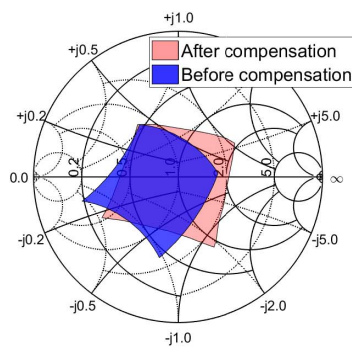

(a)

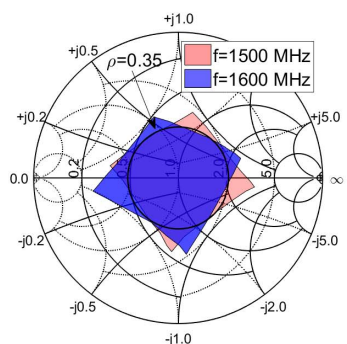

(b)
Fig. 4. Coverage area of the variable impedance mapped on the Smith chart when the bias currents $I_{2}$ and $I_{3}$ vary in the range of $[0,40] \mathrm{mA}$. Measurement results (a) before and after including the compensation inductor and (b) at different frequencies after including the compensation.

$K \in[60,100]$ and $x \in[0.7,1]$, the achievable region of $\rho$ showed no significant variation.

\section{CONCLUSION}

A new variable impedance termination consisting of two polarized diodes was studied, implemented, and measured. In good agreement with the theoretical model, the prototype can produce reflection coefficients with complete phase coverage within the circle of $\rho=0.35$ over the range of 1.5-1.6 GHz. Considering other designs that achieve higher coverage area with full phase, our approach presents a smaller number of diodes resulting in lower power consumption and simpler control of the desired impedance value. These features are well suited for power-sensitive applications [11] and N-port-based circuits [6].

\section{REFERENCES}

[1] K.-O. Sun, M. K. Choi, and D. V. D. Weide, "A PIN diode controlled variable attenuator using a 0-dB branch-line coupler," IEEE Microw. Wireless Compon. Lett., vol. 15, no. 6, pp. 440-442, Jun. 2005.

[2] J. K. Hunton and A. G. Ryals, "Microwave variable attenuators and modulators using PIN diodes," IRE Trans. Microw. Theory Techn., vol. 10, no. 4, pp. 262-273, Jul. 1962.

[3] M. Tsuji, T. Nishikawa, K. Wakino, and T. Kitazawa, "Bi-directionally fed phased-array antenna downsized with variable impedance phase shifter for ISM band," IEEE Trans. Microw. Theory Techn., vol. 54, no. 7, pp. 2962-2969, Jul. 2006.

[4] H. M. Nemati, C. Fager, U. Gustavsson, R. Jos, and H. Zirath, "Design of varactor-based tunable matching networks for dynamic load modulation of high power amplifiers," IEEE Trans. Microw. Theory Techn., vol. 57, no. 5, pp. 1110-1118, May 2009.

[5] J. Li et al., "Design of a tunable low-frequency and broadband radar absorber based on active frequency selective surface," IEEE Antennas Wireless Propag. Lett., vol. 15, pp. 774-777, 2015.

[6] Y. Zhao, C. Viereck, J. F. Frigon, R. G. Bosisio, and K. Wu, "Direct quadrature phase shift keying modulator using six-port technology," Electron. Lett., vol. 41, no. 21, pp. 1180-1181, Oct. 2005.

[7] J. Osth, O. Owais, M. Karlsson, A. Serban, S. Gong, and P. Karlsson, "Direct carrier six-port modulator using a technique to suppress carrier leakage," IEEE Trans. Microw. Theory Techn., vol. 59, no. 3, pp. 741-747, Mar. 2011.

[8] B. M. Albinsson, H. Guo, M. Schoon, and H. Vickes, "A new programmable load for noise parameter determination," IEEE Trans. Microw. Theory Techn., vol. 39, no. 2, pp. 216-223, Feb. 1991.

[9] T. Pochiraju, V. F. Fusco, J. Francey, and H. Schmassmann, "Low-power variable impedance load/tuning unit," Electron. Lett., vol. 44, no. 21, pp. 1258-1259, Oct. 2008.

[10] B.-J. Jang, I.-B. Yom, and S.-P. Lee, "An enhanced PIN diode model for voltage-controlled PIN diode attenuator," in Proc. 33rd Eur. Microw. Conf., vol. 1. Oct. 2003, pp. 231-234.

[11] P. V. Nikitin, K. V. S. Rao, and R. D. Martinez, "Differential RCS of RFID tag," Electron. Lett., vol. 43, no. 8, pp. 431-432, Apr. 2007. 